\title{
Gabapentin and pregabalin misuse
}

\author{
Journal of the Ceylon College of Physicians, 2017, 48, 59-60
}

Misuse is defined as use of a substance for a purpose not consistent with legal or medical guidelines, such as taking another person's medication or taking a higher dosage than prescribed. Prescription drug misuse is a problem, especially psychoactive drugs such as opioids, benzodiazepines and more recently gabapentinoids. Current estimates suggest $1 \%$ of the general population misuse gabapentin, as do $15-22 \%$ of people who misuse opioids, and $40-65 \%$ of people with gabapentinoid prescriptions. Pregabalin may have a higher misuse potential than gabapentin due to its rapid absorption and faster onset of action. In the UK, gabapentin and pregabalin prescribing has increased by $350 \%$ and $150 \%$ over 5 years, respectively. In 2016, gabapentin was the $10^{\text {th }}$ most commonly prescribed medication in the United States. This trend is likely due to avoidance of opioid analgesics, despite the use of gabapentin and pregabalin for non-neuropathic pain being unlicensed and ineffective. Patients inappropriately prescribed gabapentin or pregabalin may not benefit and may be exposed to potential harms, which broadly fall into two categories. First, by causing an adverse drug reaction or toxicity, e.g. central nervous system effects (headache, visual disturbance, drowsiness, agitation, delirium, lethargy); cardiac effects (tachycardia, bradycardia); disturbances in muscle control and movement, and gastrointestinal symptoms. Second, gabapentin or pregabalin prescription may result in drug misuse or diversion (the transfer of any legally prescribed substance from the individual for whom it was prescribed to another person for illicit use).

Gabapentinoids are considered to be effective for neuropathic pain. Neuropathic pain can be very difficult to treat with only some $40-60 \%$ of people achieving partial relief. Neuropathic pain is pain caused by damage or disease affecting the somatosensory nervous system ${ }^{1}$. Neuropathic pain may be associated with abnormal sensations called dysaesthesia or pain from normally non-painful stimuli (allodynia). It may have continuous and/or episodic (paroxysmal) components. The latter resemble stabbings or electric shocks. Common qualities include burning or coldness, "pins and needles" sensations, numbness and itching. Neuropathic pain may be divided into peripheral neuropathic pain, central neuropathic pain, or mixed (peripheral and central) neuropathic pain. Gabapentin was first licensed as an adjunctive treatment for epilepsy in 1993 and later approved for treatment of diabetic neuropathy and post herpetic neuralgia. However off-label prescribing of gabapentin increased in 1990s due to extensive marketing campaigns in violation of US Federal regulations promoting it for use in other pain conditions, and psychiatric disorders. Pregabalin, the newer product was approved for diabetic neuropathy and post herpetic neuralgia in 2004 and generic preparations of both these are available. In Sri Lanka too these too drugs are now freely available and the drug market is flooded with so many generic brands. Though we have no published data available, it appears that gabapentin and pregabalin are widely used mostly for off label indications. It is not uncommon to see these used as first line for chronic back pain, head ache and other musculoskeletal pains.

Both gabapentin and pregabalin are recommended by the National Institute for Health and Care Excellence for neuropathic pain. Cochrane reviews provide a similar conclusion with regard to their efficacy, noting 3 or 4 out of 10 participants achieved a greater than 50\% reduction in neuropathic pain with gabapentin, compared with 1 or 2 out of 10 for placebo. Prescribers should be aware that over half of those treated with gabapentin do not have worthwhile pain relief but may instead experience adverse effects. Similar issues affect pregabalin, a treatment which will substantially benefit only a minority of patients with neuropathic pain. Direct evidence of benefit only exists in diabetic neuropathy and herpetic neuralgia. Extrapolation of presumed benefit from this narrow subset of patients may not reflect 'real world' benefit for other causes of neuropathic pain. Existing evidence for the use of gabapentinoids in chronic lower back pain demonstrates the risk of adverse effects without any clear 
benefit. In a recent randomised controlled trial of pregabalin for acute and chronic sciatica the conclusions were that pregabalin did not significantly reduce the intensity of leg pain, but the incidence of adverse events were much higher in the treatment group.

We as prescribers must be conscious of the potential to do harm, both to our patients and society as a whole. Therefore we must carefully select the patients who will benefit from these drugs. FDA has approved gabapentin and pregabalin for post herpetic neuralgia, pregabalin for fibromyalgia and neuropathic pain associated with diabetes or spinal cord injuries. So it is time to tackle the problem of overuse of gabapentinoids by limiting their use to approved indications.

\section{References}

1. Goodman CW, Brett AS. Gabapentin and Pregabalin for pain - Is increased prescribing a cause for concern? NEJM 2017; 377: 411-13.

2. Morrison EE, Sandilands EA, Webb DJ. Gabapentin and pregabalin: do the benefits outweigh the harms? J $R$ Coll Physicians Edinb 2017; 47: 310-3.

3. Shanthanna H, Gilron I, Rajarathinam M, et al. Benefits and safety of gabapentinoids in chronic low back pain: A systematic review and meta-analysis of randomized controlled trials. PLoS Med 2017; 14: e1002369.

4. Mathieson Stephanie, Chiro M, Maher CG, et al. Trial of Pregabalin for acute and chronic sciatica 2017; 376: 1111-20.

5. Spence D. Bad medicine: gabapentin and pregabalin. BMJ 2013; 347: f6747.

\section{Saman B Gunatilake}

Co Editor

JCCP 